\title{
Effect of pulmonary exacerbations treated with oral antibiotics on clinical outcomes in cystic fibrosis
}

\author{
Sanja Stanojevic, ${ }^{1}$ Alexandra McDonald, ${ }^{1}$ Valerie Waters, ${ }^{2}$ Sarah MacDonald, ${ }^{1}$ \\ Eric Horton, ${ }^{1}$ Elizabeth Tullis, ${ }^{3,4}$ Felix Ratjen ${ }^{1}$
}

- Additional material is published online only. To view please visit the journal online (http://dx.doi.org/10.1136/ thoraxinl-2016-208450).

'Division of Respiratory Medicine, Department of Pediatrics, The Hospital for Sick Children, University of Toronto Toronto, Ontario, Canada 2Division of Infectious Diseases, Department of Pediatrics, The Hospital for Sick Children, University of Toronto, Toronto, Ontario, Canada

${ }^{3}$ Division of Respirology, Keenan Research Centre of Li Ka Shing Knowledge Institute, Toronto, Ontario, Canada

${ }^{4}$ Department of Medicine, St. Michael's Hospital, University of Toronto, Toronto, Ontario, Canada

Correspondence to Dr Felix Ratjen, Division of Respiratory Medicine, The Hospital for Sick Children, 555 University Avenue, Toronto, ON M5G 1X8, Canada; Felix. Ratjen@sickkids.ca

SS and AM contributed equally.

Received 9 February 2016 Revised 27 July 2016 Accepted 30 July 2016 Published Online First 18 August 2016

\section{ABSTRACT \\ Background Despite extensive knowledge regarding the effect of pulmonary exacerbations treated with intravenous antibiotics on clinical outcomes in cystic fibrosis (CF), there is little known about the role of milder pulmonary exacerbations treated with oral antibiotics (OPEX).}

Methods This was a retrospective cohort study of patients with CF followed at the Hospital for Sick Children and St. Michael's Hospital from 2009 to 2014. We evaluated the effect of oPEx on short-term clinical outcomes as the proportion of oPEx events in which $100 \%$ or $90 \%$ of baseline $\mathrm{FEV}_{1} \%$ predicted was recovered at the end of treatment. We then examined the association of the number of oPEx events in the past 12 months on lung function ( $\mathrm{FEV}_{1} \%$ predicted) and nutritional status (body mass index (BMI) z-score) using a mixed-effects model.

Results There were a total of 2608 oPEx events in 570 subjects during the study period. In over half $(53.4 \%)$ of oPEx events, lung function was already at $90 \%$ or higher of baseline $\mathrm{FEV}_{1}$ at the initiation of oral antibiotic therapy and $82 \%$ were at $90 \%$ or higher of baseline $\mathrm{FEV}_{1}$ at follow-up. In individuals with $\mathrm{CF}$, one or more oPex events in the previous 12 months were associated with decreased $\mathrm{FEV}_{1}$ compared with 12 months periods without oPex events. When the cumulative effect of oPExs on lung function was examined over the entire study period, patients with six or more oPEx events had the steepest rate of $F E V_{1}$ decline. oPEx events were not associated with changes in BMI.

Conclusions oPEx events are associated with shortterm loss of $\mathrm{FEV}_{1}$ and have a negative effect on lung function over time.

\section{INTRODUCTION}

Patients with cystic fibrosis (CF) experience episodes of acute worsening of respiratory symptoms, referred to as pulmonary exacerbations. In epidemiological research studies, hospitalisation or the use of intravenous antibiotics for respiratory causes is commonly used to define a severe pulmonary exacerbation. $^{1}{ }^{2}$ Severe exacerbations requiring intravenous antibiotics are associated with decreased lung function over time, ${ }^{34}$ and a quarter to a third of patients with CF do not recover their baseline $\mathrm{FEV}_{1}$ after an exacerbation and may be at risk of never regaining this lung function. ${ }^{5}$ When assessed longitudinally, approximately half of the decline in $\mathrm{FEV}_{1}$ in patients with CF is associated with these episodes of severe exacerbations. ${ }^{7}$ Despite extensive

\section{Key messages}

What is the key question?

- Although the impact of pulmonary exacerbations treated with intravenous antibiotics in patients with cystic fibrosis (CF) has been well described, there is little known about the role of milder pulmonary exacerbations treated with oral antibiotics on short lung function recovery and long-term FEV 1 decline in CF.

\section{What is the bottom line?}

- Pulmonary exacerbations treated with oral antibiotics were associated with short-term loss of $\mathrm{FEV}_{1}$ at the time of an exacerbation and an increasing number of exacerbation events were found to be independently associated with $\mathrm{FEV}_{1}$ decline.

\section{Why read on?}

- This study describes the impact of pulmonary exacerbations treated with oral antibiotics in a large adult and paediatric CF population over a 6-year period using repeated lung function and nutritional measurements, demonstrating the cumulative negative effect of these events on lung function over time.

knowledge regarding the effect of pulmonary exacerbations treated with intravenous antibiotics on clinical outcomes in $\mathrm{CF}$, there is less known about the role of milder pulmonary exacerbations treated with oral antibiotics in the CF population.

In current clinical practice, physicians often prescribe oral antibiotics to treat less severe pulmonary exacerbations. While pulmonary exacerbations treated with oral antibiotics may be classified as milder and do not lead to hospital admission, given their frequency, they may be associated with longterm clinical outcomes, especially in children. Only one study has examined the use of oral antibiotics to treat pulmonary exacerbations. Wagener et $a l^{8}$ demonstrated that pulmonary exacerbations treated with oral antibiotics have a negative effect on $\mathrm{FEV}_{1}$, but they did not define the impact on recovery of baseline lung function or long-term $\mathrm{FEV}_{1}$ decline. The objective of this study was therefore to analyse the effect of pulmonary exacerbations treated with oral antibiotics on short-term and long-term clinical outcomes in the Toronto CF population. 


\section{METHODS}

\section{Study design and data collection}

This was a retrospective cohort study of individuals with CF followed at the Hospital for Sick Children and St. Michael's Hospital (Toronto, Ontario, Canada) from 2009 to 2014. Data for this study were extracted from the Toronto CF Database housed at the Hospital for Sick Children. This encounter-based database prospectively collects information from all paediatric and adult CF subjects from every visit including sputum microbiology, medications and pulmonary function testing. ${ }^{9}$ Subjects are included in this database if they have a confirmed diagnosis of CF based on (1) the presence of clinical features consistent with CF or (2) a positive family history for CF and either two documented sweat chloride values $>60 \mathrm{mEq} / \mathrm{L}$ measured by quantitative pilocarpine iontophoresis test, genetic testing showing two CF-causing mutations or a nasal potential difference consistent with $\mathrm{CF}^{10}$ Specific information on antibiotic treatment and indication for their use was obtained through a systematic review of medical records at the Hospital for Sick Children and St. Michael's Hospital by two of the study investigators (SM and $\mathrm{EH}$ ). Antibiotics prescribed over the phone to patients who are not seen in clinic were captured in this dataset in the form of written notes included in the medical chart. If there was mention of an antibiotic course prescribed by a primary care physician in the clinic letter, this information was also included in the dataset. This study was approved by the Research Ethics Board at the Hospital for Sick Children (REB\# 1000013759) and St. Michael's Hospital (REB\# 09-087c).

\section{Study definitions}

A pulmonary exacerbation was defined as a clinical event treated with antibiotics for a respiratory cause. ${ }^{11}$ For each event, patients received either oral antibiotic treatment for a pulmonary exacerbation (oPEx) or intravenous antibiotic treatment for a pulmonary exacerbation (iPEx) based on physician assessment. During a single exacerbation, patients may have been treated with both oral and intravenous antibiotics; these events were classified as iPExs. Patients may have also experienced multiple exacerbations during the study period. The median time between consecutive exacerbations treated with oral antibiotics was 150 days (IQR 63-324 days), $<5 \%$ of all events had fewer than 10 days between oral antibiotic treatments. Similarly, the median time between consecutive events treated with oral and intravenous antibiotics was 56 days (IQR 21-184 days), $<5 \%$ of events had fewer than 4 days between treatments. Therefore, events separated by more than 1 day were classified as separate exacerbations. All analyses were also repeated with distinct events separated by 14 days. Events treated with inhaled antibiotics were not classified as pulmonary exacerbations as it is not standard practice to treat exacerbations with inhaled antibiotics alone at the Hospital for Sick Children or St. Michael's Hospital. Episodes treated with antifungal or antimycobacterial drugs were not included in the study.

The following exclusion criteria were applied for the pulmonary exacerbation events identified from 2009 to 2014 in the Toronto CF Database (figure 1): (1) active allergic bronchopulmonary aspergillosis (ABPA) as defined by the use of steroids for the treatment of ABPA as recorded in the clinicians' notes, ${ }^{12}(2)$ events treated with $>180$ days of antibiotics (representing chronic antibiotic therapy), (3) antibiotic treatment duration $<7$ days or (4) antibiotic treatment duration $>28$ days (7-28 days represented $80 \%$ of oral antibiotic treatment courses (see online supplementary figure S1)), (5) any events after lung transplantation and (6) $<2$ pulmonary function tests $(\mathrm{PFTs})$ during the study period.

The effect of oPEX events was measured on two main outcomes: $\mathrm{FEV}_{1}$ and body mass index (BMI). $\mathrm{FEV}_{1}$ values were adjusted for sex, age, height and ethnicity using Global Lung Function Initiative reference equations and standardised as per cent predicted values. ${ }^{13}$ BMI Z-scores were calculated according to sex and age using Centers for Disease Control and Prevention (CDC) Growth Charts. ${ }^{14}$ For patients older than 19 years, BMI Z-scores were calculated by extrapolating from the growth charts at age 19 .

\section{Statistical analyses}

Initial response to antibiotic treatment

To evaluate the effect of oPEx events on short-term clinical outcomes, we determined the proportion of oPEx events in which $100 \%$ or $90 \%$ of per cent predicted $\mathrm{FEV}_{1}\left(\mathrm{FEV}_{1} \%\right)$ at baseline was recovered at the end of oral antibiotic treatment. ${ }^{12}$ CIs were

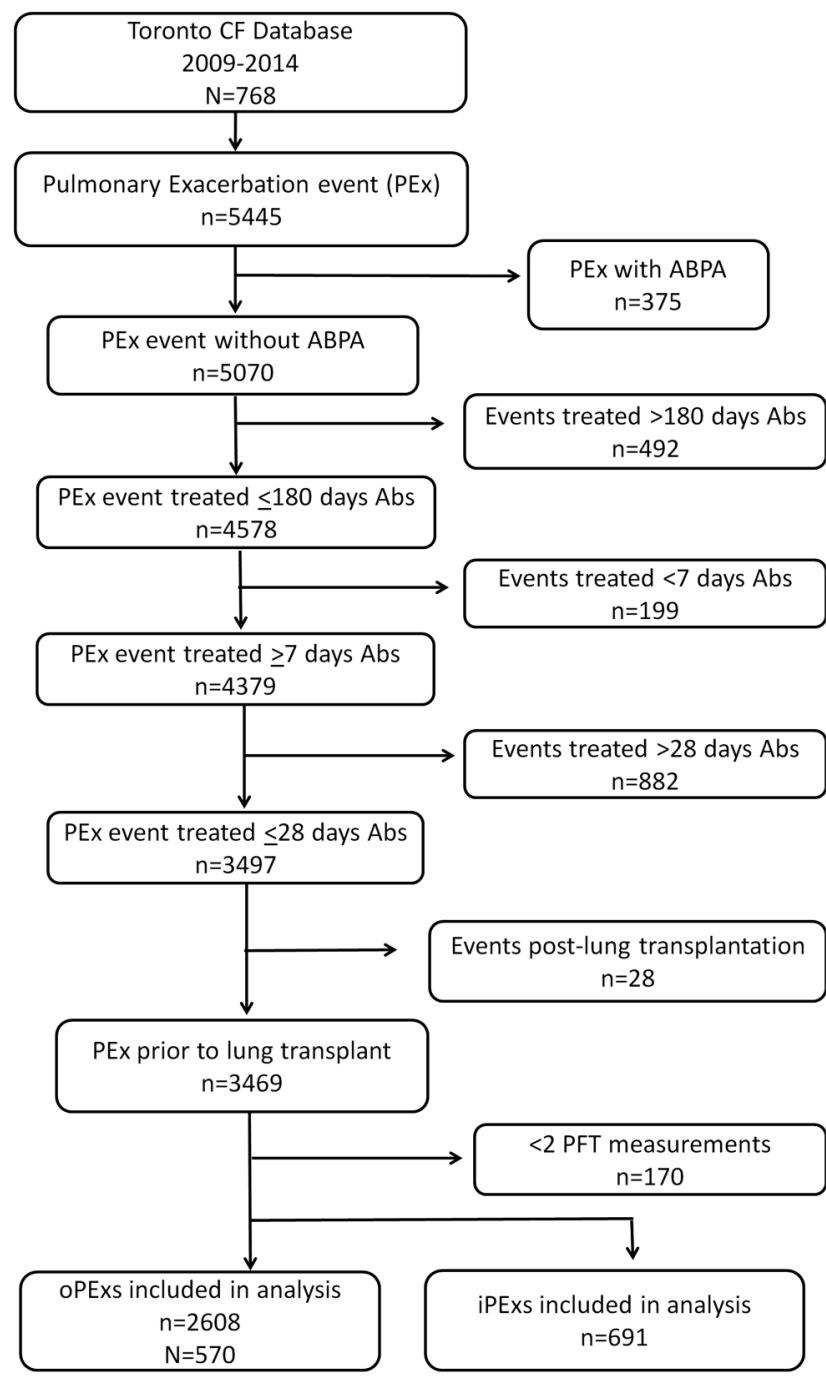

Figure 1 CONSORT diagram showing selection of study population from Toronto CF Database for pulmonary exacerbations treated with antibiotics (oPEx or iPEx) during the study period from 2009 to 2014. ABPA, allergic bronchopulmonary aspergillosis; Abs, antibiotics; CF, cystic fibrosis; iPEx, pulmonary exacerbations treated with intravenous antibiotics; oPEx, pulmonary exacerbations treated with oral antibiotics; PFT, pulmonary function test; $n$, number of pulmonary exacerbations; $\mathrm{N}$, number of subjects. 
estimated from a mixed-effects model to account for the repeated events in a single patient.

Both outcomes ( $\mathrm{FEV}_{1} \%$ predicted or BMI Z-score) were analysed at baseline, start of oPEx treatment (day 0), end of oPEx treatment and follow-up. Baseline was defined as the best value $\left(\mathrm{FEV}_{1} \%\right.$ predicted or BMI Z-score) in the 6 months prior to the exacerbation, ${ }^{5}$ where the values were restricted to stable visits (ie, did not overlap with treatment with either oral or intravenous antibiotics). Day 0 was defined as the measurement closest to start of treatment, within a window of \pm 4 days from start of treatment. End of treatment values were defined as the measurement closest to end of treatment, within a window of \pm 4 days from end of treatment. Follow-up values were defined as the best value in the 3 months following the end of treatment, ${ }^{5}$ where the value did not overlap with treatment with either oral or intravenous antibiotics.

Long-term impact on lung function and nutritional status

To examine the association of oPEx with (1) lung function $\left(\mathrm{FEV}_{1} \%\right.$ predicted) and (2) nutritional (BMI Z-score), we constructed a mixed-effects model, with random intercepts, random slopes (age) and an exchangeable correlation structure to adjust for the correlated nature of repeated outcome measurements within the same subject. The primary explanatory variable was the cumulative number of oPEx events in the previous 12 months (categorised as none, 1 or $\geq 1$ ) which was treated as a time-varying coefficient. Potential time-independent covariates included sex, pancreatic insufficiency, age at diagnosis ( $\leq 2$ years, $>2$ years), functional class of CF transmembrane conductance regulator mutation (class I-III, class IV-V, unknown), baseline PFT and BMI measurements (defined as the first recorded measurement in the study period not overlapping an antibiotic treatment period) and follow-up time in the study. The following covariates were treated as time-varying variables: age at lung function measurement, number of iPExs events in the previous 24 months (rather than 12 months given the low frequency of events, categorised as none; 1 or $\geq 1$ ), CF-related diabetes (CFRD) and age group (based on the age of the patient at lung function measurement; paediatric $\leq 18$ years of age and adult $>18$ years of age). In additional microbiology, results were treated as time-varying variables and included chronic infection with Pseudomonas aeruginosa ${ }^{15}$ and any positive respiratory tract culture in the 12 months prior to the outcome measurement for Stenotrophomonas maltophilia, Burkholderia cepacia complex, Staphylococcus aureus, Haemophilus influenzae, Aspergillus species, methicillin-resistant $S$. aureus (MRSA) infection or Achromobacter xylosoxidans.

Using a stepwise approach, we first examined the relationship between the outcome and age, and adjusted for each patient's follow-up time. Subsequently potential covariates were entered into the model, adjusted for age and follow-up time, independently to determine which factors were significantly associated with $\mathrm{FEV}_{1}$ or BMI ( $\mathrm{p}$ value $<0.15$ ). Variables were then maintained in the multivariable model if they were independently associated with the outcome or if they changed the coefficient for the effect of oPEx by more than 10\%. A backward elimination approach was used to select the variables in the final multivariable model.

\section{RESULTS}

\section{Patient characteristics}

From 2009 to 2014, 768 patients were followed in the Toronto CF database; of these, 570 subjects matched the inclusion criteria (figure 1). Overall, pulmonary symptoms were treated with oral antibiotics (oPEx) nearly four times more often than were treated with intravenous antibiotics (iPExs) during the study period (2608 vs 691). Seventy-one per cent of the CF population $(n=549)$ had at least one oPEx during the study period. The baseline characteristics of the study population $(\mathrm{N}=570)$ are presented in table 1 .

The study population had a median age of 21 years (IQR 11.5-32.9 years) and a median baseline $\mathrm{FEV}_{1} \%$ predicted of 72\% (IQR 51.9-89.2\%); the most common bacteria found in respiratory cultures was $S$. aureus. The median follow-up time for the study population was 5.5 years (IQR 3.8-5.7 years), and there was a median of 4 (IQR 2-7) oPEx events per patient during the study period. One-third of the study population had six or more oPEx episodes during the study period. The annual rate of oPEx was greater in paediatric patients (median 1.1/ patient/year (IQR 0.5-1.7) compared with adult patients (median 0.8/patient/year (0.4-1.3).

Table 1 Characteristics of the study population

\begin{tabular}{lc}
\hline & Total subjects \\
$\mathrm{N}=570$
\end{tabular}

*At each subject's first observation in the study period.

tBased on a positive culture or $P$. aeruginosa culture status (never, intermittent, chronic, as per Leed's criteria) in the 12 months prior to entry into the study cohort; eight patients were missing microbiology data.

BMI, body mass index; CFRD, cystic fibrosis-related diabetes; CFTR, CF transmembrane conductance regulator; $\mathrm{FEV}_{1} \%$ pred, $\mathrm{FEV}_{1} \%$ predicted; $\mathrm{MRSA}$, methicillin-resistant $S$. aureus. 


\section{Treatment response to oral antibiotics}

Relative to baseline lung function, $\mathrm{FEV}_{1}$ was -6.9 percentage points (95\% CI 6.1 to $7.7, \mathrm{~N}=368, \mathrm{n}=626$; $\mathrm{p}<0.001$ ) lower at the start of the oPEx, -3.6 percentage points $(95 \%$ CI 2.4 to 4.7, $\mathrm{N}=194, \mathrm{n}=24 ; \mathrm{p}<0.001)$ lower at the end of treatment and -0.88 percentage points $(95 \%$ CI 0.4 to $1.4, \mathrm{~N}=433$, $\mathrm{n}=1025 ; \mathrm{p}=0.001)$ lower at the follow-up visit. The percentage of oPEx events in which lung function was at $\geq 90 \%$ of baseline $\mathrm{FEV}_{1}$ or at $\geq 100 \%$ of baseline $\mathrm{FEV}_{1}$ at day 0 (start of treatment), end of treatment and follow-up is illustrated in figure 2. The distribution of the relative change from baseline to start of treatment for all oPEx events is shown in online supplementary figure S2. Of note, in over half of oPEx events, lung function was already at $90 \%$ or higher of baseline $\mathrm{FEV}_{1}$ at the initiation of oral antibiotic therapy and $82 \%$ were at $90 \%$ or higher of baseline $\mathrm{FEV}_{1}$ at follow-up. The proportion of oPEx events with lung function at $100 \%$ or greater of baseline $\mathrm{FEV}_{1}$ was much lower at every time point (figure 2). The response rates were similar when a 14-day interval was used to define distinct events. We also examined the response to oPEx events within different age groups (see online supplementary figure S3); none of the observed differences between the age groups were statistically significant.

\section{Association of oPEx events and lung function and nutritional status}

We first investigated the effect of oPExs on lung function by examining the association between the number of oPEx events in the previous 12 months and $\mathrm{FEV}_{1}$. Lung function was significantly decreased if patients had one or more oPEx events in the 12 months prior to the $\mathrm{FEV}_{1}$ measurement compared with 12 month periods without oPEx events (table 2-model 1). This effect was maintained after adjusting for the number of iPEx events in the 24 months prior to the lung function measurement (table 2-model 2). After adjusting for other timevarying factors (CFRD, Aspergillus infection, MRSA infection), lung function was reduced in patients with oPEx events, but the decrease was not statistically significant (table 2-model 3). Neither oPEx nor iPEx events were associated with lower BMI Z-scores in this analysis (see online supplementary table S1A).

We then examined the cumulative effect of oPExs on lung function decline during the entire study period. The overall rate

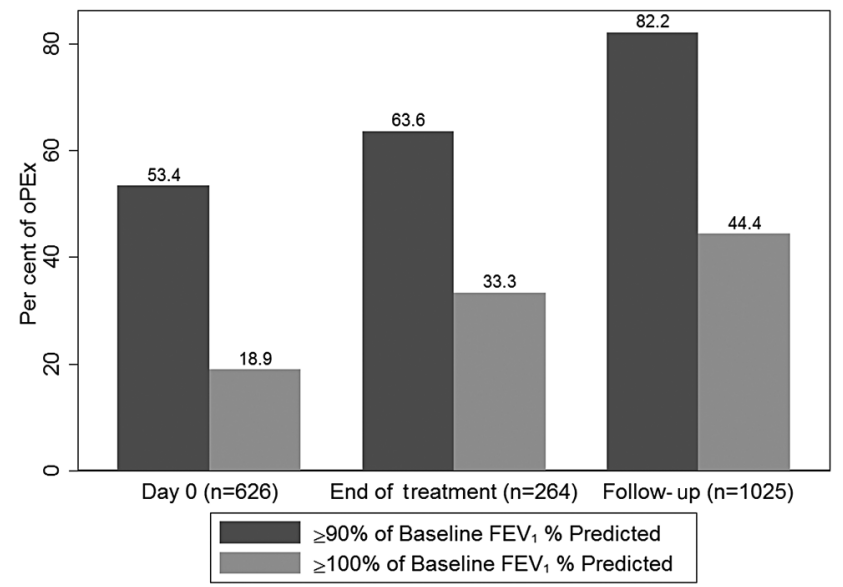

Figure 2 The per cent of oPEx events with $\geq 90 \%$ (dark grey bars) and $\geq 100 \%$ (light grey bars) of baseline $\mathrm{FEV}_{1} \%$ predicted at day 0 $(n=626)$, end of antibiotic treatment $(n=264)$ and at follow-up after oral antibiotic treatment for a pulmonary exacerbation $(n=1025)$. oPEx, pulmonary exacerbations treated with oral antibiotics. of $\mathrm{FEV}_{1}$ decline for the study population was $-1.1 \%$ per year (95\% CI -1.2 to 1.05 ). Patients in the tertile with the greatest number of oPEx events had the steepest rate of lung function decline during the study period (table 3-model 1; figure 3). This was also observed after adjustment for the other factors associated with lung function decline (number of iPEx events, age group, gender, age at diagnosis, pancreatic status, CFRD, baseline $\mathrm{FEV}_{1} \%$ predicted and infection with $H$. influenzae, Aspergillus species, A. xylosoxidans) (table 3-model 2). The results were similar in the sensitivity analysis using 14 days to separate events. In addition, the pattern of lung function decline was similar when the analysis was stratified by paediatric and adult patients (see online supplementary figure S4). For BMI, in all three oPEx groups, there was a decline over time with age; however, when adjusted for the above variables, the rate of decline was close to 0 in all three groups (see online supplementary table S1B).

\section{DISCUSSION}

In this study, we systematically measured the effects of pulmonary exacerbations treated with oral antibiotics on short-term and long-term clinical outcomes in a paediatric and adult CF population. We found that a significant proportion of patients treated with oral antibiotics for increasing respiratory symptoms did not recover baseline lung function and that oPEx events were associated with decreased lung function in patients with CF.

There is only one other study that has investigated the effects of oral antibiotic treatment for pulmonary exacerbations on lung function in CF. Wagener et $a l^{8}$ used 3 years of data from the Epidemiologic Study of Cystic Fibrosis to analyse almost 50000 pulmonary exacerbations. Their primary objective was to characterise treatment patterns of CF pulmonary exacerbations with a secondary focus on clinical outcomes. Wagener et al found that oral antibiotic use was very prevalent, especially in younger patients with less severe disease, and represented the most common method of antibiotic administration $(73 \%$ of exacerbations), a finding similar to that of the present study. The authors also found that oral antibiotic treatment was associated with a smaller absolute and relative increase in $\mathrm{FEV}_{1}$ compared with intravenous therapy, but they did not take into consideration the initial magnitude of lung function change associated with the exacerbation. Our data demonstrated that in most oPEx events (>50\%), lung function did not drop by more than $10 \%$ from baseline levels. The lack of an initial drop in lung function may have contributed to the smaller absolute and relative improvements in $\mathrm{FEV}_{1}$ observed with oral compared with intravenous antibiotic treatment. Nonetheless, in nearly $20 \%$ of these milder events treated with oral antibiotics, lung function did not improve to baseline values within 3 months of treatment. While the proportion of events was smaller than that reported $(25 \%-30 \%)$ for events treated with intravenous antibiotics, ${ }^{5}$ it represented a larger absolute number of cases as oPEx events were nearly four times more common than iPEx events.

In their assessment of longer term outcomes, Wagener et al compared the best $\mathrm{FEV}_{1}$ in the 6 months following the event to the best in the 1 year prior to the event and found that $\mathrm{FEV}_{1}$ losses were similar between oPEx and iPEx events. In our encounter-based patient registry, patients had repeated lung function measurements within shorter intervals close to the exacerbation event, as well as repeated exacerbation events over a 5-year period, which allowed us to investigate how oPEx and iPEx events affect lung function over time in patients with CF. 
Table 2 The association between oPEx events in the previous 12 months and $\mathrm{FEV}_{1} \%$ predicted

\begin{tabular}{|c|c|c|c|}
\hline & $\begin{array}{l}\text { Model } 1 \\
\text { Coefficient }(95 \% \mathrm{Cl})\end{array}$ & $\begin{array}{l}\text { Model } 2 \\
\text { Coefficient }(95 \% \mathrm{Cl})\end{array}$ & $\begin{array}{l}\text { Model } 3 \\
\text { Coefficient }(95 \% \mathrm{Cl})\end{array}$ \\
\hline \multicolumn{4}{|l|}{ oPEx event in the previous 12 months } \\
\hline 0 & Ref & Ref & Ref \\
\hline 1 & $-0.37(-0.71$ to -0.04$)$ & $-0.34(-0.67$ to -0.003$)$ & $-0.30(-0.64$ to 0.03$)$ \\
\hline$\geq 2$ & $-0.52(-0.89$ to -0.15$)$ & $-0.48(-0.86$ to -0.11$)$ & $-0.33(-0.71$ to 0.04$)$ \\
\hline \multicolumn{4}{|l|}{ iPEx event in the previous 24 months } \\
\hline 0 & Ref & Ref & Ref \\
\hline 1 & $-0.63(-1.06$ to -0.19$)$ & $-0.56(-0.99$ to -0.13$)$ & $-0.47(-0.91$ to -0.04$)$ \\
\hline$\geq 2$ & $-0.22(-0.79$ to 0.35$)$ & $-0.14(-0.71$ to 0.44$)$ & $-0.04(-0.61$ to 0.54$)$ \\
\hline Increasing agexcentred at 23 years & $-1.05(-1.19$ to -0.90$)$ & $-1.03(-1.17$ to -0.88$)$ & $-0.95(-1.09$ to 0.80$)$ \\
\hline \multicolumn{4}{|c|}{$\begin{array}{l}\text { The relationship between each predictor and } \mathrm{FEV}_{1} \text { is presented as the slopes of association }(95 \% \mathrm{Cl}) \text {. } \\
\text { Model 1: included age (centred at } 23 \text { years) and follow-up time and either oPEx or iPEx events (treated as time-varying variables). } \\
\text { Model 2: included age (centred at } 23 \text { years) and follow-up time, oPEx events in the past } 12 \text { months and iPEx events in the past } 24 \text { months both treated as time-varying variables. } \\
\text { Model 3: included age (centred at } 23 \text { years), follow-up time as well as all time-varying factors that were significant at the p<0.15 level (CFRD, Aspergillus infection, MRSA infection). } \\
\text { Negative coefficients represent decreasing FEV }{ }_{1} \text { and positive coefficients represent increasing FEV } \text {. }_{1} \text {. } \\
\text { Significant results are bold if } p<0.05 \text {. } \\
\text { CFRD, cystic fibrosis-related diabetes; MRSA, methicillin-resistant Staphylococcus aureus. }\end{array}$} \\
\hline
\end{tabular}

When the effect of oPExs on lung function was examined over a short time frame (12 months), patients with one or more episodes had decreased $\mathrm{FEV}_{1}$ compared with periods when they did not have any oPEx events. This effect was independent of more severe iPEx events but was influenced by other factors such as bacterial infection. This is not surprising given the number of factors known to influence CF lung disease. However, when the cumulative effect of oPExs was analysed over a longer time period, the negative impact on repeated oPEx events on $\mathrm{FEV}_{1}$ decline was evident, indicating that they are clinically relevant measures in individuals with CF.

Given the contribution of oPEx events on both short-term and long-term clinical outcomes in patients with CF, the results of our study raise a number of questions. The first is how to capture the treatment of milder exacerbation events that typically occur as an outpatient and are frequently not included in registry data. The second is how to treat patients with frequent, repeated oPEx events who may warrant more intensive treatment to prevent negative consequences.

The results of our study also have relevance with respect to the choice of outcome measures for clinical trials. Traditionally, pulmonary exacerbations in clinical trials have been defined by the use of intravenous antibiotics to treat major respiratory symptoms such as a decrease of $\geq 10 \%$ in $\mathrm{FEV}_{1}$, haemoptysis or radiographic changes. ${ }^{16}$ Randomised controlled studies of dornase alfa ${ }^{16}$ and hypertonic saline ${ }^{17}$ in individuals with CF have demonstrated reduction in the number of exacerbations with treatment using this definition. However, in a study of oral azithromycin in children with CF uninfected with $P$. aeruginosa, subjects presenting with exacerbations were more likely to have milder symptoms such as cough, rather than significant drops in lung function, which were treated with oral rather than intravenous antibiotics. ${ }^{18}$ Follow-up analysis of this randomised, double-blind controlled trial did not show a difference in the mean change in $\mathrm{FEV}_{1}$ between patients with no exacerbation compared with those with at least one symptom-based exacerbation in the 6-month follow-up period. ${ }^{19}$ However, the short follow-up time may have missed the long-term effects observed in the 5-year observation period of the current study. Recent interventional trials of inhaled antibiotics in patients with $\mathrm{CF}$ have demonstrated benefit in terms of reduction in milder exacerbations, defined as the use of antipseudomonal antibiotics administered by any route. ${ }^{20-22}$ With the long follow-up time in our study (over 5 years), we were able to demonstrate that repeated oPEx events have an impact on long-term lung function decline which supports the concept of including these events into pulmonary exacerbation definitions used in clinical trials.

The current study has several limitations. First, due to its retrospective nature, our data lacked specific symptoms associated with pulmonary exacerbations. We defined an oPEx based on a clinician's decision to treat with oral antibiotics which may

Table 3 Cumulative effect of total number of oPEx events during the study period and lung function decline

\begin{tabular}{|c|c|c|}
\hline & $\begin{array}{l}\text { Model } 1 \text { slope } \\
\text { Coefficient }(95 \% \mathrm{CI})\end{array}$ & $\begin{array}{l}\text { Model } 2 \text { slope } \\
\text { Coefficient }(95 \% \mathrm{CI})\end{array}$ \\
\hline $\mathrm{FEV}_{1} \%$ predicted decline with age $0-3$ oPEx events* & $-1.04(-1.15$ to -0.92$)$ & $-0.31(-0.40$ to -0.23$)$ \\
\hline $\mathrm{FEV}_{1} \%$ predicted decline with age $4-6$ oPEx events* & $-1.09(-1.21$ to -0.97$)$ & $-0.40(-0.51$ to -0.29$)$ \\
\hline $\mathrm{FEV}_{1} \%$ predicted decline with age $>6$ oPEx events* & $-1.24(-1.38$ to -1.11$)$ & $-0.75(-0.87$ to -0.62$)$ \\
\hline \multicolumn{3}{|c|}{$\begin{array}{l}\text { The relationship between each predictor and } \mathrm{FEV}_{1} \text { is presented as the slopes of association }(95 \% \mathrm{Cl}) \text {. } \\
{ }^{*} \text { Estimated by fitting stratified models for each tertile }(0-3,3-6,6+) \text { of the cumulative number of oPEx events during the study period. Negative coefficients represent decreasing } \mathrm{FEV}_{1} \\
\text { and positive coefficients represent increasing } \mathrm{FEV}_{1} \text {. } \\
\text { Model 1: included age and time in study. } \\
\text { Model 2: included age and time in study as well cumulative number of iPEx events, age group (paediatric vs adult), gender, age at diagnosis, pancreatic status, CFRD, baseline } \mathrm{FEV}{ }_{1} \% \\
\text { predicted and infection with } \mathrm{H} \text {. influenzae, Aspergillus species and } A \text {. xylosoxidans. } \\
\text { Significant results are bold if } \mathrm{p}<0.05 \text {. } \\
\text { CFRD, cystic fibrosis-related diabetes. }\end{array}$} \\
\hline
\end{tabular}




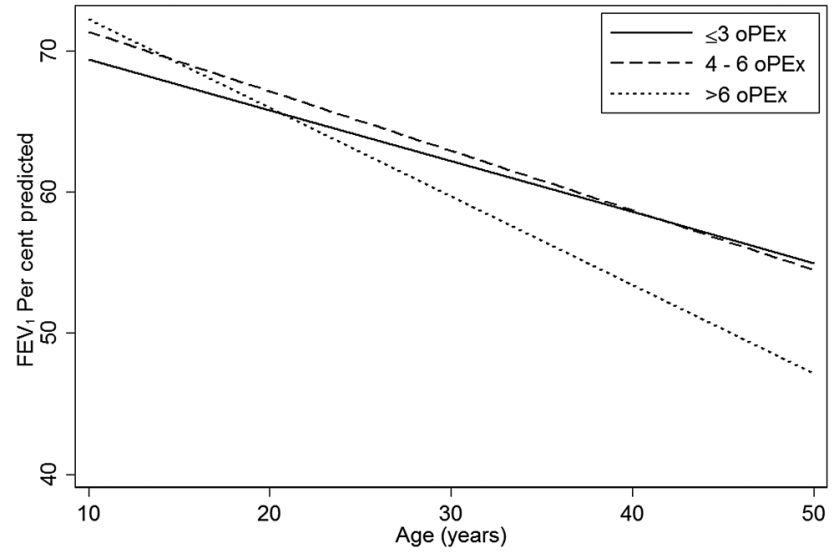

Figure 3 Change in $\mathrm{FEV}_{1} \%$ predicted based on cumulative number of oPEx events during the study period: $\leq 3$ (solid line) ( $50 \%$ of oPExs), 4-6 (hatched line) (25\% of oPExs) and $>6$ (dotted line) (25\% of oPExs). oPEx, pulmonary exacerbations treated with oral antibiotics.

have introduced an indication bias. Not all exacerbation events will come to medical attention and given that there is no standard protocol for the treatment of exacerbations at our centres, there is likely to be variability in the decision to prescribe antibiotics between different clinicians. Our primary analysis defined distinct events as those where treatment was separated by 1 day. Since treatments within 14 days may be related to the same pulmonary event, we repeated all of the analysis using 14 days between treatments to define distinct events; the results were remarkably similar. Furthermore, while a $10 \%$ change in $\mathrm{FEV}_{1}$ is routinely used as a marker of a precipitous drop in lung function indicating worsening clinical status, the normal repeatability of $\mathrm{FEV}_{1}$ is likely to be different for patients with milder disease compared with severe disease. Therefore, it is difficult to conclude whether an observed change in $\mathrm{FEV}_{1}$ is a consequence of intrinsic variability or a true measure of lung function change in this study population, for whom lung function measures were not always available at every time point for every subject. This may have introduced a potential selection bias for those who did get repeat pulmonary function testing as they may have had specific patient characteristics. To estimate the cumulative effect of repeated oPEx events, we did not have a large enough negative control group given the limited number of subjects with zero oPExs during the study period. Furthermore, we could not investigate the sequence in which oPEx and iPEx events occur, whether one is a precursor of the other. Finally, as an observational study, we cannot conclude causality based on the results, only associations.

In conclusion, oral antibiotic therapy for respiratory symptoms is very common in individuals with $\mathrm{CF}$, especially in younger patients, and is frequently triggered by factors other than acute drops in lung function. However, although these events may appear milder, like exacerbations treated with intravenous antibiotics, oPEx events are associated with loss of $\mathrm{FEV}_{1}$ compared with baseline and have a cumulative effect on lung function over time. These events represent clinically relevant study outcome measures for interventional trials in children with CF with milder lung disease.
Contributors All authors contributed to the design, data collection and analysis and writing of the manuscript.

Funding This study was funded by an unrestricted grant from Vertex Pharmaceuticals. AM was funded by a Summer Studentship from CF Canada.

Competing interests None declared.

Ethics approval Hospital for Sick Children, St Michael's Hospital.

Provenance and peer review Not commissioned; externally peer reviewed.

\section{REFERENCES}

1 Ellaffi M, Vinsonneau C, Coste J, et al. One-year outcome after severe pulmonary exacerbation in adults with cystic fibrosis. Am J Respir Crit Care Med 2005; 171:158-64.

2 Goss CH, Burns JL. Exacerbations in cystic fibrosis. 1: epidemiology and pathogenesis. Thorax 2007;62:360-7.

3 de Boer $\mathrm{K}$, Vandemheen $\mathrm{KL}$, Tullis $\mathrm{E}$, et al. Exacerbation frequency and clinical outcomes in adult patients with cystic fibrosis. Thorax 2011;66:680-5.

4 Konstan MW, Morgan WJ, Butler SM, et al. Risk factors for rate of decline in forced expiratory volume in one second in children and adolescents with cystic fibrosis. J Pediatr 2007:151:134-9, 9.e1.

5 Sanders DB, Bittner RC, Rosenfeld M, et al. Failure to recover to baseline pulmonary function after cystic fibrosis pulmonary exacerbation. Am J Respir Crit Care Med 2010;182:627-32.

6 Waters V, Atenafu EG, Salazar JG, et al. Chronic Stenotrophomonas maltophilia infection and exacerbation outcomes in cystic fibrosis. J Cyst Fibros 2012;11:8-13.

7 Waters V Stanojevic S, Atenafu EG, et al. Effect of pulmonary exacerbations on long-term lung function decline in cystic fibrosis. Eur Respir J 2012;40:61-6.

8 Wagener JS, Rasouliyan L, VanDevanter DR, et al. Oral, inhaled, and intravenous antibiotic choice for treating pulmonary exacerbations in cystic fibrosis. Pediatr Pulmonol 2013;48:666-73.

9 Kerem $\mathrm{E}$, Reisman J, Corey $\mathrm{M}$, et al. Prediction of mortality in patients with cystic fibrosis. N Engl J Med 1992;326:1187-91.

10 Farrell PM, Rosenstein BJ, White TB, et al. Guidelines for diagnosis of cystic fibrosis in newborns through older adults: cystic Fibrosis Foundation consensus report. J Pediatr 2008;153:S4-14.

11 Waters V, Yau Y, Prasad S, et al. Stenotrophomonas maltophilia in cystic fibrosis: serologic response and effect on lung disease. Am J Respir Crit Care Med 2011;183:635-40.

12 Waters V, Stanojevic $S$, Klingel M, et al. Prolongation of antibiotic treatment for cystic fibrosis pulmonary exacerbations. J Cyst Fibros 2015;14:770-6.

13 Quanjer PH, Stanojevic S, Cole TJ, et al. Multi-ethnic reference values for spirometry for the 3-95-yr age range: the global lung function 2012 equations. Eur Respir J 2012;40:1324-43.

14 Kuczmarski RJ, Ogden CL, Guo SS, et al. 2000 CDC Growth Charts for the United States: methods and development. Vital Health Stat 11 2002;246:1-190.

15 Lee TW, Brownlee KG, Conway SP, et al. Evaluation of a new definition for chronic Pseudomonas aeruginosa infection in cystic fibrosis patients. J Cyst Fibros 2003;2:29-34.

16 Fuchs HJ, Borowitz DS, Christiansen DH, et al. Effect of aerosolized recombinant human DNase on exacerbations of respiratory symptoms and on pulmonary function in patients with cystic fibrosis. The Pulmozyme Study Group. N Engl J Med 1994;331:637-42.

17 Elkins MR, Robinson M, Rose BR, et al. A controlled trial of long-term inhaled hypertonic saline in patients with cystic fibrosis. $N$ Engl J Med 2006:354:229-40.

18 Anstead M, Saiman L, Mayer-Hamblett N, et al. Pulmonary exacerbations in CF patients with early lung disease. J Cyst Fibros 2014;13:74-9.

19 Ratjen F, Saiman L, Mayer-Hamblett N, et al. Effect of azithromycin on systemic markers of inflammation in patients with cystic fibrosis uninfected with Pseudomonas aeruginosa. Chest 2012;142:1259-66.

20 Assael BM, Pressler T, Bilton $\mathrm{D}$, et al. Inhaled aztreonam lysine vs. inhaled tobramycin in cystic fibrosis: a comparative efficacy trial. J Cyst Fibros 2013:12:130-40.

21 Konstan MW, Flume PA, Kappler M, et al. Safety, efficacy and convenience of tobramycin inhalation powder in cystic fibrosis patients: The EAGER trial. J Cyst Fibros 2011;10:54-61.

22 Stuart Elborn J, Geller DE, Conrad D, et al. A phase 3, open-label, randomized trial to evaluate the safety and efficacy of levofloxacin inhalation solution (APT-1026) versus tobramycin inhalation solution in stable cystic fibrosis patients. J Cyst Fibros 2015; 14:507-14 